УДК 622.692

DOI: 10.31660/0445-0108-2019-3-102-111

Усовершенствование методики оценки технического состояния линейной части магистральных газопроводов

\author{
А. А. Разбойников ${ }^{1 *}$, Н. С. Барсуков ${ }^{2}$ \\ ${ }^{1}$ Тюменский индустриальный университет, г. Тюмень, Россия \\ ${ }^{2}$ АО «СибурТюменьГаз», г. Нижневартовск, Россия \\ *e-mail: razbojnikovaa@tyuiu.ru
}

Аннотация. Оценка технического состояния линейной части магистрального газопровода сегодня является одной из важнейших задач эксплуатации трубопроводов. Однако многие методики оценки дают лишь частичное представление о техническом состоянии и не учитывают условия прокладки трубопровода. В данной работе рассматривается усовершенствование методики оценки технического состояния магистральных газопроводов.

Ключевые слова: оценка технического состояния; ремонт магистральных газопроводов; категории прокладки газопроводов

\title{
Improving the methodology for technical assessment of the linear part of main gas pipelines
}

\author{
Aleksandr A. Razboynikov ${ }^{1 *}$, Nikolay S. Barsukov ${ }^{2}$ \\ ${ }^{I}$ Industrial University of Tyumen, Tyumen, Russia \\ ${ }^{2}$ SiburTyumenGaz JSC, Nizhnevartovsk, Russia \\ *e-mail: razbojnikovaa@tyuiu.ru
}

Abstract. Today evaluation of the technical assessment of the linear part of main gas pipeline is one of the most important tasks of pipeline operation. However, many assessment methodologies provide only a partial picture of the technical condition and don't take into account the conditions for pipeline laying. This article discusses the improvement of methods for technical assessment of main gas pipelines.

Key words: technical assessment; repair of main gas pipelines; categories of gas pipelines

\section{Введение}

Капитальный ремонт линейной части магистральных газопроводов (ЛЧМГ) является одной из наиболее важных задач обеспечения эксплуатационной надежности Единой системы газоснабжения (ЕСГ) [1]. За последнее десятилетие его ежегодные объемы увеличились в 5 раз. В настоящее время доля газопроводов, работающих на давлениях ниже проектных, достигает 14 \%. Основные методы ремонта ЛЧМГ: масштабная переизоляция газопроводов; ремонт участков газопроводов, подверженных коррозии и стресс-коррозии; ремонт газопроводов, потерявших устойчивость и изменивших проектное положение. 
Протяженность магистральных газопроводов и отводов газотранспортной сети ПАО «Газпром» [2] по состоянию на конец 2016 года на территории России составила 171,4 тыс. км. На рисунке 1 представлена информация в процентах о структуре магистральных газопроводов группы ПАО «Газпром» по возрасту на территории России.

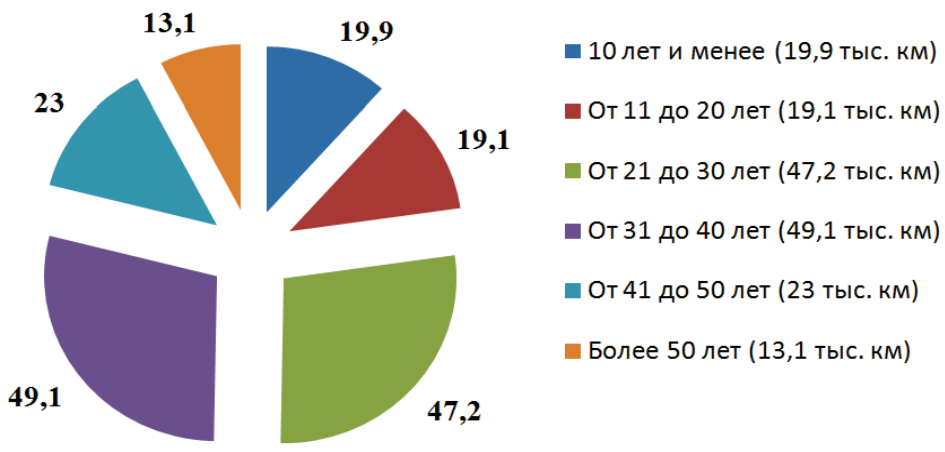

Рис. 1. Возрастной состав магистральных газопроводов газотранспортной системы ПАО «Газпром»

Увеличение объемов ремонтных работ, рост количества труб с дефектами, не подлежащими ремонту в трассовых условиях, требуют решения вопроса о необходимости усовершенствования методики оценки технического состояния линейной части магистральных трубопроводов. Данная необходимость обусловлена также и техническим состоянием газотранспортной системы России.

\section{Объект исследования}

Таким образом, из представленных выше положений сформулирован вывод о том, что объектом исследования в данной работе выступает методика оценки технического состояния линейной части магистральных газопроводов.

Существующая система оценки опасности дефектов и приоритетности вывода газопровода в ремонт (ВРД 39-1.10-001-99 ${ }^{1}$ ), применяемая ПАО «Газпром», не учитывает категорий участка газопроводов. Предлагаемая в данной статье методика математического моделирования оценки технического состояния магистральных газопроводов позволит производить анализ технического состояния магистральных газопроводов и принимать решение о выводе в ремонт газопровода с учетом категории прокладки и состава оборудования, применяемого на данном участке.

\section{Основные методы исследования}

Первым этапом предлагаемой методики является оценка степени влияния предлагаемых параметров на принятие решения. Данная оценка будет осуществляться с помощью метода попарных сравнений (например, параметров, функций, элементов и т. д.) - это наиболее точный и надежный метод выявления предпочтений. Идея метода состоит в том, что попарно сравниваются каждые два объекта, и определяется первенство одного из них. Принцип действия метода парных сравнений рассмотрим на примере.

${ }^{1}$ ВРД 39-1.10-001-99. Руководство по анализу результатов внутритрубной инспекции и оценке опасности дефектов. - Введ. 1999-05-01. - М.: ИРЦ Газпром, 1999. - 24 с. 
Принятие решения на основе алгоритма Саати [3] выполняется в следующем порядке. Экспертом заполняется матрица парных сравнений размером $\mathrm{N}$ x N, где $\mathrm{N}$ - количество альтернатив. Матрица заполняется по правилам, приведенным в таблице 1 .

таблица 1

Правила заполнения матрицы парных сравнений

\begin{tabular}{|c|l|}
\hline $\mathrm{X}_{\mathrm{ij}}$ & \multicolumn{1}{|c|}{ Значение } \\
\hline 1 & i-я и ј-я альтернативы примерно равноценны \\
\hline 3 & i-я альтернатива немного предпочтительнее $\mathrm{j}$-й \\
\hline 5 & i-я альтернатива предпочтительнее $\mathrm{j}-и ̆$ \\
\hline 7 & i-я альтернатива значительно предпочтительнее $\mathrm{j}$-й \\
\hline 9 & i-я альтернатива явно предпочтительнее $\mathrm{j}$-й \\
\hline
\end{tabular}

Если i-я альтернатива менее предпочтительна, чем j-я, то указываются обратные оценки $(1 / 3,1 / 5,1 / 7,1 / 9)$. Могут использоваться промежуточные оценки $(2,4,6,8$ и 1/2, 1/4, 1/6, 1/8); например, если і-я альтернатива совсем немного лучше $\mathrm{j}$-й, то можно использовать оценку $\mathrm{X}_{\mathrm{ij}}=2$ (тогда $\mathrm{X}_{\mathrm{ji}}=1 / 2$ ). На главной диагонали ставятся единицы. Пример заполнения матрицы представлен в таблице 2.

Таблица 2

Пример заполнения матрицы

\begin{tabular}{|c|c|c|c|c|c|c|c|c|c|}
\hline \multirow{2}{*}{ Критерии } & \multicolumn{3}{|c|}{ Сравнение } & \multicolumn{3}{|c|}{ Нормированные оценки } & \multirow{2}{*}{ сумма } & \multirow{2}{*}{ вес } & \multirow{2}{*}{$\%$} \\
\hline & $\mathrm{A} 1$ & $\mathrm{~A} 2$ & A3 & A1 & $\mathrm{A} 2$ & A3 & & & \\
\hline A1 & 1 & 5 & 9 & 1 & 5 & 9 & 15 & 0,70 & 69,57 \\
\hline $\mathrm{A} 2$ & $1 / 5$ & 1 & 4 & 0,2 & 1 & 4 & 5,2 & 0,24 & 24,12 \\
\hline A3 & $1 / 9$ & $1 / 4$ & 1 & 0,11 & 0,25 & 1 & 1,36 & 0,06 & 6,31 \\
\hline \multicolumn{7}{|c|}{ Общая сумма матрицы } & 21,56 & & 100 \\
\hline
\end{tabular}

После оценки способов расставляются числовые значения приведенных сравнений. Затем по каждому способу рассчитывается общая сумма, и находится общая сумма матрицы. После чего определяется вес каждого способа посредством деления суммы каждого элемента на общую сумму матрицы. Математически формулы будут выглядеть следующим образом:

$$
\begin{gathered}
\frac{\sum A_{i}}{\sum A_{\text {оби }}} \\
\sum A_{i}=A_{i j_{1}}+A_{i j_{2}}+A_{i j_{3}} \ldots+A_{i j_{n}}, \\
\sum_{i=1}^{n} A_{\text {оби }}=\sum A_{1}+\sum A_{2} \ldots+\sum A_{n} .
\end{gathered}
$$

Для определения важности каждого элемента формулы (1) необходимо их умножить на $100 \%$, так будет видно, насколько один параметр значительнее другого в процентном соотношении.

$$
\frac{\sum A_{i}}{\sum A_{\text {оби }}} \times 100 \% .
$$

Bторым этапом предлагаемой методики будет нанесение границ предлагаемых параметров на ось координат. По оси ординат откладываются порядковые номера предлагаемых параметров, то есть от 1 до 9. Ось абсцисс представляет собой процентное выполнение параметров, то есть вся методика берется 
за 100 процентов. На оси абсцисс откладываются параметры сравнения с учетом степени влияния данных параметров на общее состояние системы.

Третьим этапом методики является расставление баллов в соответствии с результатами внутритрубной диагностики используемого оборудования на данном газопроводе и диспетчерской задачи.

Четвертым этапом методики является построение графиков по данным параметрам с учетом технического состояния магистрального газопровода.

$\mathrm{C}$ помощью программы Microsoft Excel строим графики зависимостей выполнения критериев от весового значения критериев. Для нахождения функциональной зависимости необходимо аппроксимировать получившиеся графики с помощью построения полиномиальных линий тренда для каждого газопровода.

Для построения линий тренда воспользуемся функцией автоматического поиска линий тренда Microsoft Excel по типу полинома. После нахождения линий тренда с помощью Microsoft Excel находим функциональные зависимости процентного выполнения критериев, с указанием величины достоверности аппроксимации $\left(\mathrm{R}^{\wedge} 2\right)$.

Для определения наилучшего из имеющихся способов построим две дополнительные кривые. Первая кривая - это идеальное выполнение всех заданных условий, вторая кривая - это абсолютное невыполнение заданных условий предложенных критериев. Полученные кривые задают диапазон возможного изменения критериев.

Полученные кривые необходимо сравнить. Наиболее удобным методом сравнения будет нахождение площадей описанных кривых. Следовательно, задача сравнения будет сведена к нахождению и сравнению площадей найденных функций с помощью нахождения определенного интеграла. Поскольку необходимо произвести оценку только площади полученных кривых, то пределы интегрирования будут от 0 до 100, то есть на всей прямой. Таким образом, в общем виде интеграл будет находиться по формуле (5)

$$
S=\int_{0}^{100} y(x) d x,
$$

где $S$ - площадь подынтегральной функции; от 0 до 100 - диапазон интегрирования; $y(x)$ - функция линий тренда по каждому способу.

Для нахождения интеграла подынтегральную функцию необходимо разбить на отрезки с малым шагом равным $\Delta$. После этого найти площади каждого отрезка по всей длине подынтегральной функции. Нахождение площади на каждом отрезке будет находиться по формуле (6).

$$
S=\frac{(a+b)}{2} \cdot h,
$$

где $S$ - площадь; $b$ - основания трапеции; $h-$ высота трапеции.

В данном случае значения основания $a-$ это значения $y$ при значении $x$, а значения $b-$ значения $y$ при значении $x+\Delta$. После замен формула нахождения площади на каждом заданном отрезке с шагом $\Delta$ примет вид

$$
S=S_{i}+\Delta \frac{(y(x)+y(x+\Delta 0))}{2},
$$

где $S$ - общая площадь фигуры; $s_{i}$ - площадь предыдущего отрезка; $y(x)$ - величина 1-го основания трапеции; $y(x+\Delta)$ - величина 2-го основания трапеции; $\Delta-$ величина шага и высота трапеции $=0,001$. 


\section{Экспериментальная часть}

Для эффективной и комплексной оценки технического состояния магистральных газопроводов необходимо разработать систему, учитывающую несколько критериев. За основу возьмем ВРД 39-1.10-001-99 ${ }^{2}$.

Для оценки технического состояния линейной части газопровода необходимо выбрать ряд параметров, по которым будет проводиться анализ газопровода [4]. Выбранные параметры для оценки технического состояния газопроводов представлены в таблице 3. Количественные показатели параметров представлены в таблице 4.

таблица 3

Параметры оценки технического состояния линейной части магистрального газопровода ${ }^{3}$

\begin{tabular}{|c|l|}
\hline Обозначение & \multicolumn{1}{c|}{ Описание параметра } \\
\hline П1 & Количество опасных дефектов на единицу длины участка \\
\hline П2 & Отношение давлений, фактического к разрешенному \\
\hline П3 & Количество отказов на единицу длины трубопровода \\
\hline П4 & Вид перекачиваемого продукта \\
\hline П5 & Диаметр газопровода/ продуктопровода \\
\hline Пб & Состояние наружной изоляции \\
\hline Пг & Агрессивность грунтов \\
\hline Ко & Количество узлов, деталей единицу длины участка \\
\hline Паличие электрохимзащиты \\
\hline
\end{tabular}

Таблица 4

Количественные показатели исследуемых параметров для оценки линейной части магистральных газопроводов

\begin{tabular}{|c|c|c|c|c|c|}
\hline Параметр & \multicolumn{5}{|c|}{ Количественный показатель оценки } \\
\hline П1 & 0 & Менее 1,0 & $1,0-3,0$ & $3,0-5,0$ & Более 5,0 \\
\hline П2 & Менее 0,50 & $0,50-0,70$ & $0,70-0,85$ & $0,85-0,95$ & $0,95-1,00$ \\
\hline П3 & 0 & Менее 0,03 & $0,03-0,06$ & $0,06-0,09$ & Более 0,09 \\
\hline П4 & $\begin{array}{c}\text { Газ } \\
\text { (без КАД*) }\end{array}$ & $\begin{array}{c}\text { Газ, продукт } \\
\text { с КАД* }\end{array}$ & $\begin{array}{c}\text { Конденсат } \\
\text { без КАД* }\end{array}$ & $\begin{array}{c}\text { Жидкий этан } \\
\text { без КАД* }\end{array}$ & $\begin{array}{c}\text { ШФЛУ** } \\
\text { без КАД* }\end{array}$ \\
\hline П5 (газ.) & Менее 219 & $219-377$ & $530-720$ & $1020-1220$ & 1420 \\
\hline (продукт.) & Менее 219 & $219-273$ & $325-377$ & 530 & 720 \\
\hline П6 & Хорошее & - & Удовлетв. & - & Плохое \\
\hline П7 & Отсутст. & Низкая & Средняя & - & Высокая \\
\hline П8 & Менее 0,1 & $0,1-0,4$ & $0,4-0,7$ & $0,7-1,0$ & Более 1,0 \\
\hline П9 & Есть & - & - & - & Нет \\
\hline
\end{tabular}

Примечание. *КАД-коррозионно-активная добавка; ** ШФЛУ- -ирокая фракиия легких углеводородов; газ. - газопровод; продукт. - продуктопровод.

Параметры П1-П3, П8 определяются по формулам (8)-(11).

Формула для определения параметра П1

$$
\Pi 1=\frac{L}{k},
$$

${ }^{2}$ ВРД 39-1.10-001-99. - С.14-22.

${ }^{3}$ СТО Газпром 2-2.3-095-2007. Методические указания по диагностическому обследованию линейной части магистральных газопроводов. - Введ. 2007-08-28. - М.: ИРЦ Газпром, 2007. - 91 с. 
где $L$ - длина участка газопровода, км; $k$ - количество опасных дефектов на данном участке, шт.

Формула для определения параметра П2

$$
\Pi 2=\frac{P_{\text {факт }},}{P_{\text {разр. }}},
$$

где $P_{\text {факт }}-$ фактическое давление в газопроводе, МПа; $P_{\text {разр. }}-$ разрешенное давление в газопроводе, МПа.

Формула для определения параметра П3

$$
\Pi 3=\frac{x}{L},
$$

где $x$ - количество отказов на данном участке газопровода, шт; $L-$ длина участка газопровода, км.

Формула для определения параметра П8

$$
\Pi 8=\frac{n}{L},
$$

где $n-$ количество крановых узлов, тройников, колен и пр., шт; $L-$ длина участка газопровода, км.

Недостатком методики, представленной в ВРД 39-1.10-001-99 ${ }^{4}$, является то, что в данной методике отсутствует комплексная оценка дефектов трубопровода совместно с оценкой категорий участка трубопровода.

Существует пять различных категорий участков трубопроводов. Данные категории участков учитывают условия прокладки газопровода, геологические условия местности и назначение магистрального газопровода. Для упрощения расчетов и наглядности разрабатываемую методику представим на трех наиболее распространенных категориях участков: категория I, категория II, категория III. Чем выше категория участка, тем более сложные условия строительства и эксплуатации газопровода, тем выше должен быть осуществляемый контроль полученных дефектов.

Оценку технического состояния линейной части магистрального газопровода предлагается осуществлять с помощью начисления баллов по каждому параметру (см. табл. 3), с учетом состояния исследуемого участка (см. табл. 4), с учетом категории участка трубопровода (табл. 5).

\begin{tabular}{|c|c|c|c|c|c|c|c|c|c|c|c|c|c|c|c|}
\hline № & I & II & III & $\mathrm{I}$ & II & III & $\mathrm{I}$ & II & III & $\mathrm{I}$ & II & III & $\mathrm{I}$ & II & III \\
\hline \multirow{2}{*}{ П1 } & \multicolumn{3}{|c|}{ Нет дефектов } & \multicolumn{3}{|c|}{ Менее 1,0} & \multicolumn{3}{|c|}{$1,0-3,0$} & \multicolumn{3}{|c|}{$3,0-5,0$} & \multicolumn{3}{|c|}{ Более 5,0} \\
\hline & 0 & 0 & 0 & 11 & 6 & 1 & 12 & 7 & 2 & 13 & 8 & 3 & 14 & 9 & 4 \\
\hline \multirow{2}{*}{ П2 } & \multicolumn{3}{|c|}{ Менее 0,50} & \multicolumn{3}{|c|}{$0,50-0,70$} & \multicolumn{3}{|c|}{$0,70-0,85$} & \multicolumn{3}{|c|}{$0,85-0,95$} & \multicolumn{3}{|c|}{$0,95-1,00$} \\
\hline & 10 & 5 & 0 & 11 & 6 & 1 & 12 & 7 & 2 & 13 & 8 & 3 & 14 & 9 & 4 \\
\hline \multirow{2}{*}{ П3 } & \multicolumn{3}{|c|}{ Нет отказов } & \multicolumn{3}{|c|}{ Менее 0,03} & \multicolumn{3}{|c|}{$0,03-0,06$} & \multicolumn{3}{|c|}{$0,06-0,09$} & \multicolumn{3}{|c|}{ Более 0,09} \\
\hline & 0 & 0 & 0 & 11 & 6 & 1 & 12 & 7 & 2 & 13 & 8 & 3 & 14 & 9 & 4 \\
\hline \multirow{2}{*}{ П4 } & \multicolumn{3}{|c|}{ Газ (без КАД) } & \multicolumn{3}{|c|}{$\begin{array}{c}\text { Газ, продукт } \\
\text { с КАД }\end{array}$} & \multicolumn{3}{|c|}{$\begin{array}{c}\text { Конденсат } \\
\text { без КАД }\end{array}$} & \multicolumn{3}{|c|}{$\begin{array}{c}\text { Жидкий этан } \\
\text { без КАД }\end{array}$} & \multicolumn{3}{|c|}{ ШФЛУ без КАД } \\
\hline & 10 & 5 & 0 & 11 & 6 & 1 & 12 & 7 & 2 & 13 & 8 & 3 & 14 & 9 & 4 \\
\hline \multirow{3}{*}{ П5 } & \multicolumn{3}{|c|}{ Менее 219} & \multicolumn{3}{|c|}{$219-377$} & \multicolumn{3}{|c|}{$530-720$} & \multicolumn{3}{|c|}{$1020-1220$} & \multicolumn{3}{|c|}{1420} \\
\hline & \multicolumn{3}{|c|}{ Менее 219} & \multicolumn{3}{|c|}{$219-273$} & \multicolumn{3}{|c|}{$325-377$} & \multicolumn{3}{|c|}{530} & & 720 & \\
\hline & 10 & 5 & 0 & 11 & 6 & 1 & 12 & 7 & 2 & 13 & 8 & 3 & 14 & 9 & 4 \\
\hline
\end{tabular}

Таблица 5

\section{Начисление баллов по параметрам с учетом категорий}

${ }^{4}$ ВРД 39-1.10-001-99. - C.14-22. 
Продолжение табл. 5

\begin{tabular}{|c|c|c|c|c|c|c|c|c|c|c|c|c|c|c|c|}
\hline № & I & II & III & $\mathrm{I}$ & II & III & $\mathrm{I}$ & II & III & I & II & III & I & II & III \\
\hline \multirow{2}{*}{ П6 } & \multicolumn{3}{|c|}{ Хорошее } & \multicolumn{3}{|c|}{-} & \multicolumn{3}{|c|}{ Удовлетв. } & \multirow{2}{*}{\multicolumn{3}{|c|}{-}} & \multicolumn{3}{|c|}{ Плохое } \\
\hline & 0 & 0 & 0 & & & & 12 & 7 & 2 & & & & 14 & 9 & 4 \\
\hline \multirow{2}{*}{ П7 } & \multicolumn{3}{|c|}{ Отсутствует } & \multicolumn{3}{|c|}{ Низкая } & \multicolumn{3}{|c|}{ Средняя } & \multicolumn{3}{|c|}{-} & \multicolumn{3}{|c|}{ Высокая } \\
\hline & 0 & 0 & 0 & 11 & 6 & 1 & 12 & 7 & 2 & & & & 14 & 9 & 4 \\
\hline \multirow{2}{*}{ П8 } & \multicolumn{3}{|c|}{ Менее 0,1} & \multicolumn{3}{|c|}{$0,1-0,4$} & \multicolumn{3}{|c|}{$0,4-0,7$} & \multicolumn{3}{|c|}{$0,7-1,0$} & \multicolumn{3}{|c|}{ Более 1,0} \\
\hline & 10 & 5 & 0 & 11 & 6 & 1 & 12 & 7 & 2 & 13 & 8 & 3 & 14 & 9 & 4 \\
\hline \multirow{2}{*}{ П9 } & \multicolumn{3}{|c|}{ Есть } & \multicolumn{3}{|c|}{-} & \multicolumn{3}{|c|}{-} & \multirow{2}{*}{\multicolumn{3}{|c|}{-}} & \multicolumn{3}{|c|}{ Нет } \\
\hline & 0 & 0 & 0 & & & & & & & & & & 14 & 9 & 4 \\
\hline
\end{tabular}

Проверку данной методики проведем на сравнительном анализе трех магистральных газопроводов. Для полноты оценки каждый из представленных газопроводом рассмотрим в трех категориях, исследуемых в предлагаемой методике. Данные представлены в таблице 6.

таблица 6

Газопроводы, используемые для апробации методики

\begin{tabular}{|c|c|c|c|c|c|c|c|c|c|c|c|c|c|}
\hline \multirow{3}{*}{ 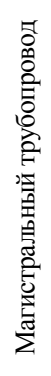 } & \multicolumn{11}{|c|}{ Описание исследуемого газопровода } & \multirow{3}{*}{ 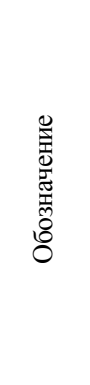 } & \multirow{3}{*}{ 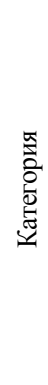 } \\
\hline & \multirow[b]{2}{*}{ 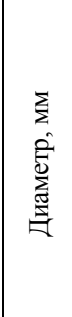 } & \multirow[b]{2}{*}{ 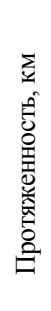 } & \multicolumn{2}{|c|}{$\begin{array}{c}\text { Давление, } \\
\text { МПА }\end{array}$} & \multirow{2}{*}{ 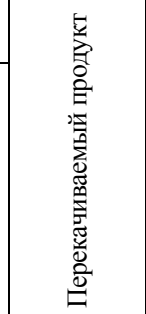 } & \multirow[b]{2}{*}{ 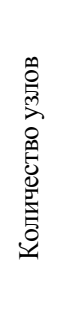 } & \multirow[b]{2}{*}{ 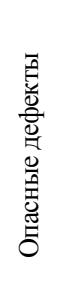 } & \multirow{2}{*}{ 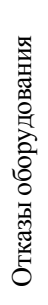 } & \multirow{2}{*}{ 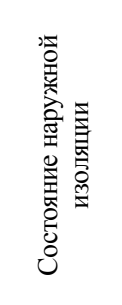 } & \multirow{2}{*}{ 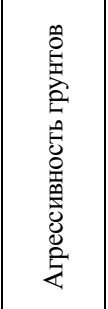 } & \multirow[b]{2}{*}{ 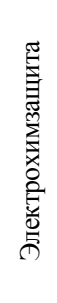 } & & \\
\hline & & & 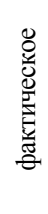 & 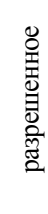 & & & & & & & & & \\
\hline \multirow{3}{*}{ 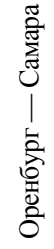 } & \multirow{3}{*}{1020} & \multirow{3}{*}{119} & \multirow{3}{*}{4,6} & \multirow{3}{*}{4,7} & \multirow{3}{*}{$\begin{array}{c}\text { Природный } \\
\text { газ } \\
\text { (без КАД) }\end{array}$} & \multirow{3}{*}{19} & \multirow{3}{*}{316} & \multirow{3}{*}{11} & \multirow{3}{*}{ Хорошее } & \multirow{3}{*}{ Низкая } & \multirow{3}{*}{+} & МГ 1.1 & III \\
\hline & & & & & & & & & & & & МГ 1.2 & II \\
\hline & & & & & & & & & & & & МГ 1.3 & I \\
\hline 节 & \multirow{3}{*}{1020} & \multirow{3}{*}{117} & \multirow{3}{*}{3,2} & \multirow{3}{*}{6,4} & \multirow{3}{*}{$\begin{array}{c}\text { Природный } \\
\text { газ } \\
\text { (без КАД) }\end{array}$} & \multirow{3}{*}{15} & \multirow{3}{*}{61} & \multirow{3}{*}{5} & \multirow{3}{*}{ Удовлетв. } & \multirow{3}{*}{ Высокая } & & МГ 2.1 & III \\
\hline 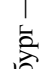 & & & & & & & & & & & - & МГ 2.2 & II \\
\hline ठे & & & & & & & & & & & & МГ 2.3 & I \\
\hline 怘 & & & & & & & & & & & & МГ 3.1 & III \\
\hline I & 377 & 176 & 5,0 & 5,5 & $\begin{array}{c}\text { ШФЛУ } \\
\text { (без КАД) }\end{array}$ & 173 & 109 & 5 & Плохое & Средняя & + & МГ 3.2 & II \\
\hline हैं & & & & & & & & & & & & МГ 3.3 & I \\
\hline
\end{tabular}

Исходя из алгоритма, представленного ранее, для определения степени влияния различных параметров на решение вывода газопровода в ремонт не- 
обходимо определить степень важности исследуемых критериев. Определение степени важности исследуемых критериев представлено в таблице 7.

Количественная оценка критериев

\begin{tabular}{|c|c|c|c|c|c|c|c|c|c|c|c|}
\hline & П1 & П2 & П3 & П4 & П5 & П6 & П7 & П8 & П9 & $\Sigma$ & $\%$ \\
\hline П1 & 1 & 3 & 4 & 7 & 4 & 8 & 3 & 6 & 9 & 45,0 & 19,2 \\
\hline П2 & 0,33 & 1 & 4 & 7 & 4 & 8 & 5 & 7 & 9 & 45,3 & 19,3 \\
\hline П3 & 0,25 & 0,25 & 1 & 6 & 4 & 6 & 3 & 5 & 9 & 34,5 & 14,7 \\
\hline П4 & 0,14 & 0,14 & 0,17 & 1 & 4 & 4 & 7 & 5 & 9 & 30,5 & 13,0 \\
\hline П5 & 0,25 & 0,25 & 0,25 & 0,25 & 1 & 8 & 6 & 4 & 9 & 29,0 & 12,4 \\
\hline П6 & 0,13 & 0,13 & 0,17 & 0,25 & 0,14 & 1 & 5 & 5 & 9 & 20,8 & 8,9 \\
\hline П7 & 0,33 & 0,20 & 0,33 & 0,14 & 0,17 & 0,20 & 1 & 5 & 9 & 16,4 & 7,0 \\
\hline П8 & 0,17 & 0,14 & 0,11 & 0,20 & 0,25 & 0,20 & 0,20 & 1 & 9 & 11,3 & 4,8 \\
\hline П9 & 0,11 & 0,11 & 0,11 & 0,11 & 0,11 & 0,11 & 0,11 & 0,11 & 1 & 1,9 & 0,8 \\
\hline
\end{tabular}

Из данной таблицы видно, какие критерии сравнения оказывают наибольшее влияние на линейную часть магистрального газопровода, а какие меньшее. Следовательно, при построении общего графика критерии, имеющие большее влияние, займут большую площадь.

Начислим баллы исследуемым газопроводам, по данным таблицы 6. Начисленные баллы представлены в таблице 8

Таблица 8

Баллы газопроводов с учетом категорий участков

\begin{tabular}{|c|c|c|c|c|c|c|c|c|c|}
\hline Обозначение & П1 & П2 & П3 & П4 & П5 & П6 & П7 & П8 & П9 \\
\hline МГ 1.1 & 3 & 4 & 4 & 0 & 3 & 0 & 0 & 3 & 0 \\
\hline МГ 1.2 & 8 & 9 & 9 & 5 & 8 & 0 & 0 & 8 & 0 \\
\hline МГ 1.3 & 13 & 14 & 14 & 10 & 13 & 0 & 0 & 13 & 0 \\
\hline МГ 2.1 & 1 & 1 & 2 & 1 & 2 & 2 & 4 & 1 & 0 \\
\hline МГ 2.2 & 6 & 6 & 7 & 6 & 7 & 7 & 9 & 6 & 0 \\
\hline МГ 2.3 & 11 & 11 & 12 & 11 & 12 & 12 & 14 & 11 & 0 \\
\hline МГ 3.1 & 2 & 0 & 3 & 4 & 2 & 4 & 2 & 4 & 4 \\
\hline МГ 3.2 & 7 & 5 & 8 & 9 & 7 & 9 & 7 & 9 & 9 \\
\hline МГ 3.3 & 12 & 10 & 13 & 14 & 12 & 14 & 12 & 14 & 14 \\
\hline
\end{tabular}

\section{Результаты эксперимента}

После начисления баллов строим графики для определения функций и нахождения интегралов. Графики «идеального» газопровода, не требующего капитального ремонта — «Міn». График газопровода, требующего срочного ремонта - «Мах». Полученные функциональные зависимости и величина достоверной аппроксимации представлены в таблице 9. 
Функциональные зависимости исследуемых газопроводов

\begin{tabular}{|c|c|c|}
\hline Обозначение & Функции линий тренда & $\mathrm{R}^{\wedge} 2$ \\
\hline МГ 1.1 & $\mathrm{y}=2 \cdot 10^{7} \mathrm{x}^{4}-3 \cdot 10^{5 \cdot} \mathrm{x}^{3}+0,0022 \mathrm{x}^{2}+0,002 \mathrm{x}+0,9671$ & 0,998 \\
\hline МГ 1.2 & $\mathrm{y}=1 \cdot 10^{7} \mathrm{x}^{4}-2 \cdot 10^{5} \mathrm{x}^{3}+0,0019 \mathrm{x}^{2}-0,0067 \mathrm{x}+0,8769$ & 0,994 \\
\hline МГ 1.3 & $\mathrm{y}==-7 \cdot 10^{8} \mathrm{x}^{4}+2 \cdot 10^{8} \mathrm{x}^{3}-0,0008 \mathrm{x}^{2}+0,0558 \mathrm{x}+0,153$ & 0,985 \\
\hline МГ 2.1 & $\mathrm{y}=9 \cdot 10^{6} \mathrm{x}^{3}-0,0008 \mathrm{x}^{2}+0,0724 \mathrm{x}+0,8579$ & 0,997 \\
\hline МГ 3.1 & $\mathrm{y}=1 \cdot 10^{7} \mathrm{x}^{4}-1 \cdot 10^{5} \mathrm{x}^{3}+0,0005 \mathrm{x}^{2}+0,0468 \mathrm{x}+0,8973$ & 0,997 \\
\hline Max & $\mathrm{y}=2 \cdot 10^{8} \mathrm{x}^{5}-7 \cdot 10^{6} \mathrm{x}^{4}+0,0007 \mathrm{x}^{3}-0,0365 \mathrm{x}^{2}+0,8826 \mathrm{x}-6,8304$ & 0,995 \\
\hline Min & $\mathrm{y}=5 \cdot 10^{9 \cdot} \mathrm{x}^{5}-1 \cdot 10^{6} \mathrm{x}^{4}+9 \cdot 10^{5 \cdot} \mathrm{x}^{3}-0,0029 \mathrm{x}^{2}+0,0818 \mathrm{x}+0,9998$ & 1 \\
\hline
\end{tabular}

После нахождения функциональных зависимостей проведем математический анализ полученных результатов, вычислив интегралы заданных функций. Результаты вычисления занесем в таблицу 10.

Таблица 10

Значения полученных интегралов

\begin{tabular}{|c|c|}
\hline Обозначение & Значение полученных интегралов \\
\hline МГ 1.1 & 490,030 \\
\hline МГ 1.2 & 387,514 \\
\hline МГ 1.3 & 387,623 \\
\hline МГ 2.1 & 406,032 \\
\hline МГ 3.1 & 440,291 \\
\hline Max & 305,749 \\
\hline Min & 595,627 \\
\hline
\end{tabular}

Обсуждение

Проведем сравнительный анализ полученных данных. Начнем с анализа газопроводов МГ 1.1, 1.2, 1.3. Данные газопроводы идентичны по составу оборудования и состоянию вспомогательных систем, главным отличием является категория прохождения газопровода. Газопровод МГ 1.1 относится к третьей категории сложности газопроводов, МГ 1.2 - ко второй категории, МГ 1.3 к первой категории. Исходя из граничных условий максимума и минимума изменений, делаем вывод о том, что газопровод с минимальным значением интеграла функции можно отнести к газопроводу, требующему срочного ремонта, то есть газопровод МГ 1.2 является первоочередным к проведению ремонта.

Следующим этапом анализа является сравнительный анализ различных газопроводов, относящихся к одной категории прокладки трубопровода (это трубопроводы МГ 1.1, 2.1, 3.1). Исходя из граничных условий максимума и минимума изменений, делаем вывод о том, что газопровод с минимальным значением интеграла функции можно отнести к газопроводу, требующему срочного ремонта, то есть газопровод МГ 2.1 является первоочередным к проведению ремонта. 


\section{Выводы}

- Учет условий прокладки важен для оценки технического состояния линейной части магистрального газопровода.

- $\quad$ Предлагаемая методика позволяет производить анализ газопроводов в одинаковых и в различных категориях прокладки.

- Предлагаемый в методике алгоритм расчета является динамическим то есть данный алгоритм позволяет добавлять, убирать, заменять параметры сравнения и изменять численные показатели в зависимости от поставленной задачи.

- Из таблицы 9 видно, что погрешность расчетов составляет до 1,5 \%.

\section{Библиографический список}

1. Сбор и подготовка нефти и газа: учеб. / Ю. Д. Земенков [и др.]. - М.: Академия, 2009. - 159 c.

2. Внутренняя сила. Годовой отчет ПАО «Газпром» за 2016 год [Электронный ресурс]. - Режим доступа: http // www.gazprom.ru.

3. Саати Т. Принятие решение. Метод анализа иерархий. - М.:Радио и связь, 1993. $278 \mathrm{c}$

4. Методологические основы научных исследований: учеб. пособие для студентов нефтегазового профиля / Под ред. Ю. Д. Земенкова. - Тюмень: Вектор Бук, 2011. - 289 с.

\section{Сведения об авторах}

Разбойников Александр Адольфович, к. т.н., доцент кафедры транспорта углеводородных ресурсов, Тюменский индустриальный университет, г. Тюмень, e-mail: razbojnikovaa@tyuiu.ru

Барсуков Николай Сергеевич, машинист технологических компрессоров АO «СибурТюменьГаз», г. Нижневартовск

\section{Information about the authors}

Aleksandr A. Razboynikov, Candidate of Engineering, Associate Professor at the Department of Transportation of Hydrocarbon Resources, Industrial University of Tyumen, e-mail: razbojnikovaa@tyuiu.ru

Nikolay S. Barsukov, Compressor Operator, SiburTyumenGaz JSC, Nizhnevartovsk 